\title{
Uncertainty of Boundary Layer Heat Budgets Computed from Wind Profiler-RASS Networks
}

\author{
Markus Furger* and C. David Whiteman \\ Pacific Northwest Laboratory, Richland, Washington \\ JAMES M. WILCZAK \\ NOAA Environmental Technology Laboratory, Boulder, Colorado
}

(Manuscript received 16 December 1993, in final form 1 July 1994)

\section{ABSTRACT}

\begin{abstract}
Uncertainties in the evaluation of the atmospheric heat budget, in which the turbulent heat fux divergence term is calculated as a residual, are investigated for a triangular array of $915-\mathrm{MHz}$ wind profilers-radio acoustic sounding systems (RASS) using a surface-integral method. A scaling analysis of the residual error heat budget equation reveals the basic characteristics and magnitudes of the uncertainties. These values are verified with a Monte Carlo simulation technique for synthetic datasets in which the triangle size is of the order of $30 \mathrm{~km}$ (meso- $\gamma$ scale). The uncertainties depend on measurement errors, atmospheric stability, mean wind speed, triangle size, and averaging time. In addition, we estimate the effects of baroclinity and mean wind divergence on the accuracy of the calculation of the heat budget.

Idealized, barotropic, and divergence-free conditions are studied to investigate the influence of various instrument accuracies on profiles of the turbulent virtual potential temperature flux divergence term. Results show that this term can be computed as a residual of the other terms with an uncertainty that varies from approximately 0.4 to $1.6 \mathrm{~K} \mathrm{~h}^{-1}$ for typical ranges of mean wind speed and stability, given current accuracies for $1-\mathrm{h}$ averages of wind profiler-RASS. Uncertainties of the remaining terms in the equation are smaller. Although the uncertainties found are of about the same magnitude as typical maximum daytime boundary layer turbulent sensible heat flux divergences, $1.2 \mathrm{~K} \mathrm{~h}^{-1}$, it is found that under favorable conditions meaningful turbulent heat flux divergences can be obtained. The computations, however, become very uncertain under conditions of strong baroclinity or wind divergence.
\end{abstract}

\section{Introduction}

The development in the last two decades of groundbased remote sensing devices such as radar wind profilers (WP) and radio acoustic sounding systems (RASS) now offers the possibility of near-real-time continuous computations of derived kinematic quantities, such as divergence and vorticity, and components of mass and heat budgets. In the past, such computations were made from arrays of three or more tethersonde or rawinsonde stations (e.g., Binder et al. 1989; Saarikivi and Puhakka 1990) but were restricted in temporal and spatial resolution by the limited frequency of balloon launches, by sonde drift, and by questionable representativeness of point measurements

\footnotetext{
* Permanent affiliation: Paul Scherrer Institute, Villigen, Switzerland.

Corresponding author address: Dr. Markus Furger, Paul Scherrer Institute, LUS/Environmental and Systems Analysis Laboratory, CH5232 Villigen PSI, Switzerland.
}

in space and time. The latter problem is particularly important for computations in the earth's atmospheric boundary layer $(A B L)$. The remote sensors measure vertical profiles of wind and temperature with resolutions of less than $1 \mathrm{~h}$ in time and on the order of $100 \mathrm{~m}$ in the vertical. Each measurement is a volume average over a sampling volume defined by the beamwidth and the length of the range gate. Usually a series of such individual measurements is averaged over a selected time interval-for example, $1 \mathrm{~h}$.

With the deployment of networks of WP-RASS in recent years, initial studies of kinematic quantities in the troposphere have been performed (Carlson and Forbes 1989; Hermes 1991). The latter study revealed a large scatter in the resulting calculations caused by profiler measurement errors and concluded that efforts must be made to improve measurement accuracy. Both studies were restricted to the analysis of wind data above the ABL. Temperature profiles from RASS are generally not available at those heights.

This study focuses on the question of whether current remote sensor measurements of wind and temper- 
ature allow the computation of volumetric heat budgets for the ABL. A surface-integral method is used to calculate the budget terms for a triangular array of WPRASS. Given values of measurement uncertainties, a Monte Carlo simulation yields information on the expected uncertainties of the calculations. The method is applied to an idealized situation.

\section{Instrument accuracies}

The maximum possible error (systematic and/or random) that can affect an observed value is commonly referred to as the accuracy of an individual measurement. For WP-RASS, we do not yet have complete knowledge of systematic errors because of difficulties in getting independent measurements describing a known state of the atmosphere. In other words, the absolute accuracy of WP-RASS has yet to be determined, and the best information available is on relative accuracies and precisions. Several investigators have presented values derived from various intercomparisons and other analyses (Strauch et al. 1987; Wuertz et al. 1988; May et al. 1989; Weber et al. 1990; Weber and Wuertz 1990; Moran et al. 1991; Neff and Wilczak 1992; Carter et al. 1992; Martner et al. 1993; Ye et al. 1993; Angevine et al. 1994). The difficulties in getting a complete picture of instrument accuracies arise from the fact that the values have been obtained under different conditions and for different types of WP-RASS. Often the results from such studies have been used to improve measurement accuracy, and hence newer publications should give better and more reliable accuracy estimates. The values depend on the type of instrument, the season, the height, the weather situation, and the sampling or averaging mode (Wuertz et al. 1988; Gaynor and Ye 1993). The 915-MHz wind profilers are now able to reach accuracies of 1 $\mathrm{m} \mathrm{s}^{-1}$ under favorable conditions. This value was used for the control simulation, and we selected additional values from a range of $0.2-3 \mathrm{~m} \mathrm{~s}^{-1}$ to cover a wider spectrum of possible accuracy values.

Assessing the true accuracy of RASS temperature measurements is difficult. Direct comparisons with rawinsonde data suggest that rms RASS errors are on the order of $1 \mathrm{~K}$. However, more detailed studies (Moran and Strauch 1994; Angevine and Ecklund 1994) show consistent RASS biases on the order of $0.5-1.0 \mathrm{~K}$ that are slowly varying functions of height. Recent theoretical analyses have largely explained these observed biases (Lataitis et al. 1993; G. Peters 1993, personal communication ). After accounting for the RASS bias errors, Angevine and Ecklund (1994) found that the rms RASS-rawinsonde temperature differences are on the order of $0.5 \mathrm{~K}$, a value close to the precision of rawinsonde measurements (Hoehne 1980). The true error of the RASS measurements is likely to be limited only by the precision of RASS, approximately $0.2 \mathrm{~K}$ (May et al. 1989) and by sampling limitations due to the finite observation time of RASS measurements. For these reasons we examined the sensitivity of our heat budget calculations to a range of RASS errors from 0.2 to $3 \mathrm{~K}$, with our control simulation using a value of $0.5 \mathrm{~K}$.

The accuracy values of $0.5 \mathrm{~K}$ and $1 \mathrm{~m} \mathrm{~s}^{-1}$ are valid for hourly averaged winds and 5-min-averaged temperatures with a $100-\mathrm{m}$ resolution. This averaging is done to reduce sampling errors and to bring the WPRASS measurements into closer agreement with the true atmospheric mean values. If individual measurements were considered, then, under the assumption that there are no systematic errors, the standard deviation of the differences between WP-RASS and the true value would have to be increased by a factor $n^{1 / 2}$, where $n$ is the number of values included in the averaging process (Strauch et al. 1984; Wuertz et al. 1988). For the $915-\mathrm{MHz}$ wind profiler, $n$ is usually less than or equal to 20 .

The accuracies of hourly averaged data also show some dependence on height caused by decreased signal-to-noise ratios, leading to a decreasing number of data points at higher levels (Strauch et al. 1987). We use height-independent values of standard deviations for our calculations. For RASS data this is supported by the results of May et al. (1989), showing opposite height dependencies for summer and winter, but a constant value for the combined dataset.

Radiation measurement errors are usually given as relative errors with a magnitude of the order of $5 \%-$ $10 \%$ (for a pyrradiometer). Assuming a surface net radiative flux value of $300 \mathrm{~W} \mathrm{~m}^{-2}$, this yields an error of $15-30 \mathrm{~W} \mathrm{~m}^{-2}$. Because the radiative flux divergence is the small difference of two large quantities, satisfactory values can only be obtained for sufficiently deep atmospheric layers (Kondratyev 1972). Hence, a resulting divergence error of 0.05 $\mathrm{K} \mathrm{h}^{-1}$, derived from an average flux error of 1.5 $\mathrm{W} \mathrm{m} \mathrm{m}^{-2}$ for a 100 - $\mathrm{m}$ layer (or $15 \mathrm{~W} \mathrm{~m}^{-2}$ for a 1000 $m$ layer), will be used as the radiation measurement error for our analysis. Typical radiative heating and cooling rates in the ABL are on the order of $0.1 \mathrm{~K} \mathrm{~h}^{-1}$ or less, except in strong temperature inversions, where locally they can reach values of up to a few kelvins per hour in extreme cases (Garratt and Brost 1981).

\section{Methods for the evaluation of the heat budget equation}

\section{a. Equations and basic assumptions}

If we assume that radiation is the only diabatic process affecting the heat budget of a volume of air, the equation for the conservation of virtual potential temperature in turbulent flow has the form 


$$
\begin{gathered}
\frac{\partial \bar{\theta}_{v}}{\partial t}+\underbrace{\nabla_{h} \cdot\left(\overline{\mathbf{v}}_{h} \bar{\theta}_{v}\right)}_{\mathrm{Bh}}+\underbrace{\frac{\partial\left(\bar{w} \overline{\theta_{v}}\right)}{\partial z}+\underbrace{\nabla \cdot\left(\overline{\mathbf{v}^{\prime} \theta_{v}^{\prime}}\right)}_{\mathrm{C}}}_{\mathrm{Bv}} \\
+\underbrace{\frac{1}{\bar{\rho} c_{p}} \nabla \cdot \overline{\mathbf{R}}}_{\mathrm{D}}=0 \quad\left(\mathrm{~K} \mathrm{~s}^{-1}\right),
\end{gathered}
$$

where $\rho$ is the density of moist air, $c_{p}$ is the specific heat capacity of moist air, $\theta_{v}$ is the virtual potential temperature, $\mathbf{v}$ is the wind vector, and $\mathbf{R}$ is the net allwave radiation vector. We will henceforth refer to (1) as to our heat budget equation, although we should keep in mind that (1) is proportional, but not equal, to changes in heat content (enthalpy; see, e.g., Pielke 1984 , p. 13). The units of kelvins per second represent a heating or cooling rate.

The heat budget of a volume $d V$ (usually a triangular slice) can be obtained by integrating (1) over the volume. Term $A$ then represents the time rate of change of heat storage. Terms $\mathrm{Bh}$ and $\mathrm{Bv}$ are the horizontal and vertical components of the mean virtual potential temperature flux divergence, and the sum of $\mathrm{Bh}$ and $\mathrm{Bv}$ will be referred to as term $\mathrm{B}$. Term $\mathrm{C}$ is the turbulent virtual potential temperature flux divergence, and term $\mathrm{D}$ is the net radiative flux divergence. The integral equation can be solved using the divergence theorem for the conversion of terms B and D into surface integral forms to obtain

$$
\begin{array}{r}
\iiint \frac{\partial \bar{\theta}_{v}}{\partial t} d V+\iint \mathbf{n} \cdot \overline{\mathbf{v}} \bar{\theta}_{v} d S+\iiint \nabla \cdot\left(\overline{\mathbf{v}^{\prime} \theta_{v}^{\prime}}\right) d V \\
+\frac{1}{\bar{\rho} c_{p}} \iint \mathbf{n} \cdot(\nabla \cdot \overline{\mathbf{R}}) d S=0,
\end{array}
$$

where $S$ is the surface of the volume, $\mathbf{n}$ is the unit vector normal to the corresponding sidewall or lid, and the density $\bar{\rho}$ is obtained by integrating the hydrostatic equation. This solution method is called the surfaceintegral method and is analogous to the line-integral method described by Davies-Jones (1993). Evaluation of (2) involved the computation of time derivatives in term $\mathrm{A}$ with a centered finite-differencing scheme, using values from the temperature profiles preceding and following the time for which the budget was evaluated. Virtual temperature measured by the RASS and corrected for vertical velocity (Weber et al. 1992) was converted to virtual potential temperature using the formula for dry static energy divided by $c_{p}$,

$$
\theta_{v}=T_{v}+\frac{g z}{c_{p}},
$$

where $T_{v}$ is virtual temperature, $g$ is gravity acceleration, and $z$ is height.

Because humidity profiles cannot be measured with the RASS, the value $c_{p d}=1005 \mathrm{~J} \mathrm{~kg}^{-1} \mathrm{~K}^{-1}$ for dry air was used in place of $c_{p}$ for moist air. Since $c_{p d} \leqslant c_{p}$, this leads to an overestimation of the values of $\theta_{v}$ in (3) by less than $0.07 \%$ for reasonable $\mathrm{ABL}$ values. The overall effect of this approximation is therefore negligible in terms of the heat budget evaluation.

Term $B$ can be calculated by summing over all sidewalls and the top and bottom lids. Horizontal wind data are directly measured with the profiler, whereas vertical wind components are calculated from horizontal wind divergence using the continuity equation.

For term $\mathrm{D}$ it is assumed that horizontal radiative flux divergences are negligible compared to vertical flux divergence, so that only contributions from the bottom and top lid are considered. Radiative flux divergences are generally not measured directly but are calculated using a radiative transfer model (dependent on measured or assumed temperature and moisture profiles). We used McKee and Whiteman's (1977) radiative transfer model calculations, which used mean climatological temperature and humidity sounding data from Grand Junction, Colorado, and typical seasonal profiles of $\mathrm{CO}_{2}, \mathrm{O}_{3}$, and aerosols to determine typical net longwave radiation profiles for the lowest $2 \mathrm{~km}$. A profile for a "dry" atmosphere was calculated by adjusting the humidities down two standard deviations. We applied this single radiative divergence profile at all times. The radiative transfer modeling did not include the effects of clouds, however, or the detailed effects of humidity and temperature fine structure in the nearground layer.

Finally, term $\mathrm{C}$ is calculated as the residual of the other terms in the heat budget. With such an approach, all errors in the other terms are projected to term $\mathrm{C}$. Nevertheless, we can expect that the uncertainties of term $\mathrm{C}$ obtained in this way will provide us with upper bounds to the true uncertainties. We will compare our results to values extracted from the scientific literature.

\section{b. Uncertainty analysis}

The evaluation of the heat budget equation is subject to uncertainties. The goal of an uncertainty analysis is to provide information on the magnitude of the total error composed of all contributions from all error sources. It can be represented in the form of confidence intervals or error bars, and we will subsequently refer to it as uncertainty or, simply, error. Davies-Jones (1993) presented analytical formulas for the estimation of errors in the derived quantities resulting from random wind-observing errors. In contrast, we have chosen a simple scaling analysis together with a Monte Carlo simulation approach to gain insight into the sensitivity of the surface-integral method.

\section{1) SCALING ANALYSIS}

To aid in our sensitivity analysis of the heat budget terms to variations in both mean atmospheric condi- 
tions and in assumed errors of the measured wind speeds and temperatures, we develop a heat budget error equation. Expressing the measured velocities and temperatures as the sum of true values and measurement errors,

$$
\begin{aligned}
& \hat{\theta}=\bar{\theta}+\delta \theta \\
& \hat{\mathbf{v}}=\overline{\mathbf{v}}+\delta \mathbf{v},
\end{aligned}
$$

where $\bar{\theta}, \overline{\mathbf{v}}$ are true mean values, and $\delta \theta, \delta \mathbf{v}$ are measurement errors. We then substitute the measured values into (1). For simplicity we assume, for the moment, horizontal homogeneity of the true wind and temperature fields. By subtracting the balance of the true heat budget, the remaining expression for the residual error (RE) in the budget becomes

$$
\begin{aligned}
\underbrace{\frac{\partial \delta \theta}{\partial t}}_{\mathrm{A}}+\underbrace{\overline{\mathbf{v}}_{h} \cdot \nabla_{h} \delta \theta}_{\mathrm{Bhm}}+\underbrace{\delta \mathbf{v}_{h} \cdot \nabla_{h} \delta \theta}_{\mathrm{Bhp}}+\underbrace{\delta w \frac{\partial \bar{\theta}}{\partial z}}_{\mathrm{Bvm}} \\
+\underbrace{\delta w \frac{\partial \delta \theta}{\partial z}}_{\mathrm{Bvp}}+\underbrace{\frac{1}{\bar{\rho} c_{p}} \frac{\partial \delta R_{z}}{\partial z}}_{\mathrm{D}}=\mathrm{RE},
\end{aligned}
$$

where $\delta w$ is found from continuity

$$
\nabla_{h} \cdot \delta \mathbf{v}_{h}+\frac{\partial \delta w}{\partial z}=0
$$

Using characteristic values of $\delta \theta=0.5 \mathrm{~K}, \delta \mathrm{v}=1$ $\mathrm{m} \mathrm{s}^{-1},|\mathrm{v}|=10 \mathrm{~m} \mathrm{~s}^{-1}, \partial \bar{\theta} / \partial z=5 \mathrm{~K} \mathrm{~km}^{-1}$, and $\delta R_{z}$ $=1.5 \mathrm{~W} \mathrm{~m}^{-2} ;$ a triangle size of $30 \mathrm{~km}$; and centered differences in time and height, we scale each of the terms in (4) to obtain the following:

$\mathrm{A}: \frac{\partial \delta \theta}{\partial t} \approx \frac{0.5 \mathrm{~K}}{2 \mathrm{~h}} \rightarrow 0.25 \mathrm{~K} \mathrm{~h}^{-1}$

$\mathrm{Bhm}: \quad \overline{\mathrm{v}}_{h} \cdot \nabla_{h} \delta \theta \approx\left(10 \mathrm{~m} \mathrm{~s}^{-1}\right)\left(\frac{0.5 \mathrm{~K}}{30 \mathrm{~km}}\right) \rightarrow 0.6 \mathrm{~K} \mathrm{~h}^{-1}$

Bhp: $\quad \delta \mathbf{v}_{h} \cdot \nabla_{h} \delta \theta \approx\left(1 \mathrm{~m} \mathrm{~s}^{-1}\right)\left(\frac{0.5 \mathrm{~K}}{30 \mathrm{~km}}\right) \rightarrow 0.06 \mathrm{~K} \mathrm{~h}^{-1}$

Bvm: $\quad \delta w \frac{\partial \bar{\theta}}{\partial z} \approx\left(0.016 \mathrm{~m} \mathrm{~s}^{-1}\right)\left(\frac{5 \mathrm{~K}}{1 \mathrm{~km}}\right) \rightarrow 0.3 \mathrm{~K} \mathrm{~h}^{-1}$

Bvp:

$$
\begin{aligned}
& \delta w \frac{\partial \delta \theta}{\partial z} \approx\left(0.016 \mathrm{~m} \mathrm{~s}^{-1}\right)\left(\frac{0.5 \mathrm{~K}}{200 \mathrm{~m}}\right) \rightarrow 0.15 \mathrm{~K} \mathrm{~h}^{-1} \\
& \mathrm{D}: \frac{1}{\bar{\rho} c_{p}} \frac{\partial \delta R_{z}}{\partial z} \approx \frac{0.0015 \mathrm{~K} \mathrm{~m} \mathrm{~s}^{-1}}{100 \mathrm{~m}} \rightarrow 0.05 \mathrm{~K} \mathrm{~h}^{-1} .
\end{aligned}
$$

We note that in the estimation of terms Bvm and Bvp, $\delta w$ has been evaluated from (5) at a height of $1000 \mathrm{~m}$.
The maximum possible value of $\delta w$ occurs when $\delta v$ errors are perfectly correlated with height, which would give $\delta w=0.033 \mathrm{~m} \mathrm{~s}^{-1}$ in the estimation of terms Bvm and Bvp. For scaling purposes we use a more realistic value of one-half the maximum value, or $\delta w=0.016$ $\mathrm{m} \mathrm{s}^{-1}$. The same characteristic values will be used later in the Monte Carlo simulations.

Here we divide the net horizontal and vertical advection terms into components dependent on mean or perturbation terms. We note that the only terms that are height dependent are Bvm and Bvp, representing the mean and perturbation vertical advection. These terms are zero at the surface, where $\delta w$ is zero, and increase with height due to the propagation of error in the calculation of $\delta w$ from (5). Clearly the second-order term Bhp is much smaller than the remaining terms, except for the radiation term.

Up to this point we have assumed that there is no mean baroclinity or divergence present. Estimates of the relative importance of these effects can be derived by including these effects in the derivation of (4). This results in two additional terms:

mean baroclinity: $\delta \mathrm{v} \cdot \nabla_{h} \bar{\theta}$

moderate baroclinity:

$$
\left(1 \mathrm{~m} \mathrm{~s}^{-1}\right)\left(\frac{1 \mathrm{~K}}{30 \mathrm{~km}}\right) \rightarrow 0.12 \mathrm{~K} \mathrm{~h}^{-1}
$$

strong baroclinity: $\left(1 \mathrm{~m} \mathrm{~s}^{-1}\right)\left(\frac{10 \mathrm{~K}}{30 \mathrm{~km}}\right) \rightarrow 1.2 \mathrm{~K} \mathrm{~h}^{-1}$

mean divergence: $\quad \bar{w} \frac{\partial \delta \theta}{\partial z}$

mean divergence:

$$
\left(0.15 \mathrm{~m} \mathrm{~s}^{-1}\right)\left(\frac{0.5 \mathrm{~K}}{200 \mathrm{~m}}\right) \rightarrow 1.4 \mathrm{~K} \mathrm{~h}^{-1}
$$

strong divergence:

$$
\left(0.45 \mathrm{~m} \mathrm{~s}^{-1}\right)\left(\frac{0.5 \mathrm{~K}}{200 \mathrm{~m}}\right) \rightarrow 4.1 \mathrm{~K} \mathrm{~h}^{-1} .
$$

It becomes evident that both effects, especially divergence, can contribute significantly to the heat budget uncertainty, becoming clearly the dominant contribution in certain situations. For our simulations, however, we will assume horizontal homogeneity and, hence, neglect these two effects.

\section{2) Monte Carlo technique}

Monte Carlo simulations give uncertainty estimates for large ensembles of "realistic" situations that are statistically scattered with a given distribution function around an unperturbed "true" value. The standard deviation of the ensemble of simulated cases corresponds 
to the measurement precision of the physical process to which the Monte Carlo technique has been applied. The Monte Carlo method provides no information on whether the unperturbed value is the true value - that is, the bias or absolute accuracy cannot be determined with this procedure. The results are interpreted in a statistical sense: the uncertainty estimate is a $68 \%$ chance to find the true value within plus or minus one standard deviation from the measured value, provided there is no systematic error in the measurement.

Monte Carlo simulations use random numbers to generate a large ensemble of synthetic datasets, which can be used as input data for the analysis technique or model to be tested. In our case, individual terms of the atmospheric heat budget equation were evaluated using assumed data profiles from an artificial network of three WP-RASS. Given a complete set of input values, the program was run to calculate the heat budget for each measurement level. After this initial run, a random perturbation was added to each of the input values, and the whole calculation was repeated, giving different results. Repeating the last step many times yielded a distribution of scattered values that can be analyzed statistically - one from which conclusions can be drawn about the sensitivity of the method to the variation of input values. The resulting standard deviations of 1000 Monte Carlo simulations define the uncertainties of the budget terms obtained using the surface-integral method.

The uncertainties of the input values were simulated by randomly sampling values from normal distributions of input variables having zero mean values and standard deviations obtained from published relative instrument accuracies for wind profilers and RASS's. A discussion of these values was given in section 2 . The random numbers were generated using procedures described by. Press et al. (1989).

An equilateral triangle with a side length of $30 \mathrm{~km}$ was selected for the simulations. This size is reasonable for meso- $\gamma$-scale studies, and the equilateral shape minimizes gradient errors that arise on triangles having a very short side (Thiébaux and Pedder 1987). The triangle is assumed to be located in ideally level terrain, allowing the use of a Cartesian $(x, y, z)$ coordinate system. For this study a vertical resolution of $100 \mathrm{~m}$ was used. Each profile consisted of 20 levels, simulating the lowest $2000 \mathrm{~m}$ of the atmosphere. The 915$\mathrm{MHz}$ RASS can reach this height under relatively calm conditions with neutral stability.

\section{Results of the simulations}

To examine the effects of various measurement errors on the uncertainty of the heat budget equation, we first performed a control simulation. This control run served as a basis for comparison with other scenarios in which one parameter at a time was varied, while all other parameters were kept fixed.

\section{a. Control run}

Our strategy for the simulations was to start with stationary and horizontally homogeneous temperature, wind, and radiation fields. In such a setting all horizontal divergences are identically zero. This run will be referred to as the control run. A $10 \mathrm{~m} \mathrm{~s}^{-1}$ wind speed was assumed at all levels, and temperature decreased linearly with height from $10^{\circ} \mathrm{C}$ at the surface to $0^{\circ} \mathrm{C}$ at $2000 \mathrm{~m}$. The radiative flux divergence was assumed to be constant throughout the model domain, with a value corresponding to a cooling rate of $1 \mathrm{~K} \mathrm{day}^{-1}$. We used standard deviation values of $0.5 \mathrm{~K}$ for temperature, 1 $\mathrm{m} \mathrm{s}^{-1}$ for wind components, and $1.5 \mathrm{~W} \mathrm{~m}^{-2}$ for radiative flux, respectively. These values, except for the radiative flux, represent current measurement accuracies for 1-h averages.

The results of the control run simulation are presented in Fig. 1. Remember that term B is simply the sum of its components $\mathrm{Bh}$ and $\mathrm{Bv}$. The mean values of the budget terms A (time rate of change of heat storage) and $B$ (potential temperature flux divergence) are close to zero, as expected. Term $\mathrm{D}$ is slightly positive, representing the value of $0.04 \mathrm{~K} \mathrm{~h}^{-1}\left(1 \mathrm{~K} \mathrm{day}^{-1}\right)$ quite well. Consequently, term $\mathrm{C}$ is slightly negative, compensating this and the other contributions. The small fluctuations are due to the finite number of Monte Carlo simulations that were performed, and these fluctuations decrease with increasing numbers of simulations. The

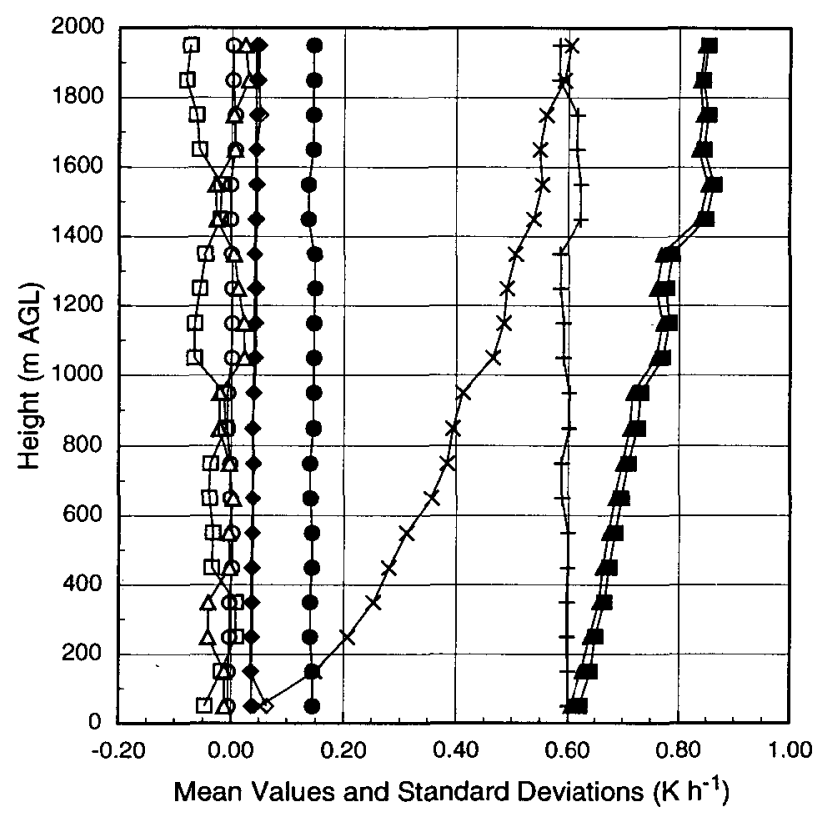

FIg. 1. Mean values (outline symbols) and standard deviations (solid symbols) of heat budget terms for the control run with assumed measurement errors of $0.5 \mathrm{~K}$ for temperature, $1 \mathrm{~m} \mathrm{~s}^{-1}$ for wind components, and $1.5 \mathrm{~W} \mathrm{~m}^{-2}$ for net radiation as functions of height AGL. Statistics computed from 1000 Monte Carlo simulations; term A (๑); term B $(\Delta)$; term Bh $(+)$; term Bv $(\times)$; term C $(\square)$; and term D $(\diamond)$. 
standard deviations of terms $\mathrm{A}$ and $\mathrm{Bh}$ are roughly constant with height, while the standard deviations of the other terms increase with height. The approximate values of the standard deviations at the surface are 0.14 $\mathrm{K} \mathrm{h}^{-1}, 0.61 \mathrm{~K} \mathrm{~h}^{-1}, 0.62 \mathrm{~K} \mathrm{~h}^{-1}$, and $0.04 \mathrm{~K} \mathrm{~h}^{-1}$ for terms $\mathrm{A}, \mathrm{B}, \mathrm{C}$, and $\mathrm{D}$, respectively. The increase with height in term $\mathrm{Bv}$ is due to propagation of error effects in the estimation of $\delta w$. In general, the scaling [ section $3 b(1)]$ describes quite well the error magnitudes for each term found in the control simulation.

The relative contributions of terms A, B, and D on term $\mathrm{C}$ for this special case can be inferred from Fig. 1. The standard deviation of term $C$ is the sum of the squares of the standard deviations of the other terms.

Carlson and Stull (1986) give values in the range from 0.08 to $2.1 \mathrm{~K} \mathrm{~h}^{-1}$ for the stable ABL for terms $A-D$, with turbulence causing the largest amount of cooling in that particular case. In subsequent sections we will focus our attention on the turbulent virtual potential temperature flux divergence (term $\mathrm{C}$ ). The results of this term can be compared with a typical maximum value of turbulent sensible heat flux divergence in a convective boundary layer of $1.2 \mathrm{~K} \mathrm{~h}^{-1}$, as obtained, for example, from a situation with a surface heat flux of $300 \mathrm{~W} \mathrm{~m}^{-2}$, an entrainment flux of $-50 \mathrm{~W} \mathrm{~m}^{-2}$, and an ABL height of $1000 \mathrm{~m}$.

\section{b. Effects of wind errors}

Figure 2 shows the results from simulations of term $\mathrm{C}$ for which only the accuracy of wind measurements has been varied. We used standard deviations of 0.2 , 1,2 , and $3 \mathrm{~m} \mathrm{~s}^{-1}$ for each wind component. The uncertainties of term $\mathrm{C}$ increase with height, and the slope of the curve is related to the wind measurement error. However, the intercepts of the curves are the same. Referring to (4), we see that the sensitivity to the horizontal wind errors enters directly through term Bhp, and also indirectly into terms Bvm and Bvp through $\delta w$. Decreasing $\delta \mathrm{v}$ below $1 \mathrm{~m} \mathrm{~s}^{-1}$ has little effect because, as seen in the control run (Fig. 1) and in our scaling analysis, contributions from terms Bhp, Bvm, and Bvp do not significantly increase the total advection term B. However, by increasing $\delta \mathrm{v}$ to $3 \mathrm{~m} \mathrm{~s}^{-1}$, the net residual error increases by a factor of 2 at a height of $1000 \mathrm{~m}$ because of the increases in terms Bvm and Bvp. This increase in the residual error above the surface would be reduced if the atmospheric stability approached neutral.

The increase with height of the standard deviation of term $\mathrm{C}$, whose shape and magnitude is dominated in this case by term B, correlates well with the increase of the standard deviation of the vertical wind component computed from mass continuity. A possible way to improve this situation would be to use the vertical wind components measured directly by the profiler. Measurement problems, however, make this approach problematic.

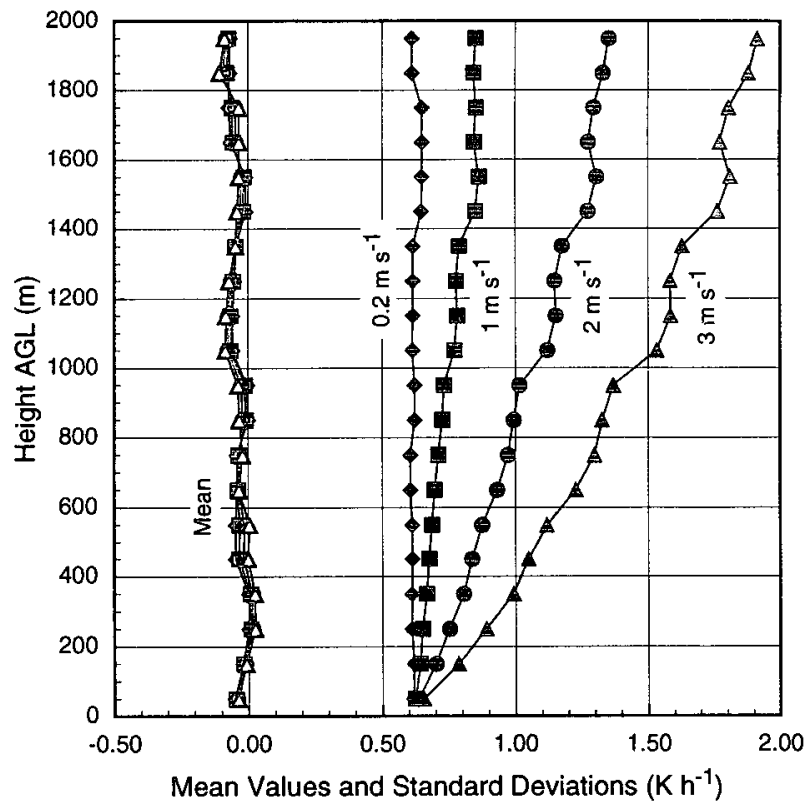

FiG. 2. Mean values (denoted as "mean," outline symbols) and standard deviations (solid symbols) of the turbulent virtual potential temperature flux divergence (term $\mathrm{C}$ ) calculated as a residual of the other terms for wind measurement errors of $0.2,1,2$, and $3 \mathrm{~m} \mathrm{~s}^{-1}$. The other measurement errors are the same as for the control run. Statistics computed from 1000 Monte Carlo simulations; $0.2 \mathrm{~m} \mathrm{~s}^{-1}$ $(\bullet) ; 1 \mathrm{~m} \mathrm{~s}^{-1}$ (毘); $2 \mathrm{~m} \mathrm{~s}^{-1}(\bullet)$; and $3 \mathrm{~m} \mathrm{~s}^{-1}(\mathbf{A})$.

\section{c. Sensitivity to mean wind}

The largest term in the control run was found to be the horizontal advection, Bh. Since our scaling analysis indicates that the dominant part of this term is Bhm (the horizontal advection due to the mean wind), and because Bhm is proportional to $|\mathbf{v}|$, we expect that the net residual error will be roughly proportional to $|\mathrm{v}|$, except for low wind speeds where other (smaller) terms become important. This behavior is verified in the Monte Carlo simulations for mean wind speeds of $0,5,10$, and $15 \mathrm{~m} \mathrm{~s}^{-1}$ (Fig. 3). We note that for $|\mathrm{v}|$ $\leqslant 5 \mathrm{~m} \mathrm{~s}^{-1}$, the residual error is less than the mean value of term $\mathrm{C}$ for many meteorological situations.

\section{d. Effects of temperature errors}

The effects of random temperature errors on the uncertainty of turbulent virtual potential temperature flux divergence are presented in Fig. 4. We performed calculations for measurement errors of $0.2,0.5,1,2$, and $3 \mathrm{~K}$. The computed standard deviations do not show a strong height dependence, in contrast to the effects of wind errors discussed previously. The shift in the magnitude of this standard deviation is proportional to the temperature measurement errors. Figure 4 indicates that an improvement in temperature accuracies to values of $0.2 \mathrm{~K}$ would decrease the resulting standard deviation of term $\mathrm{C}$ to approximately $0.4 \mathrm{~K} \mathrm{~h}^{-1}$, consid- 


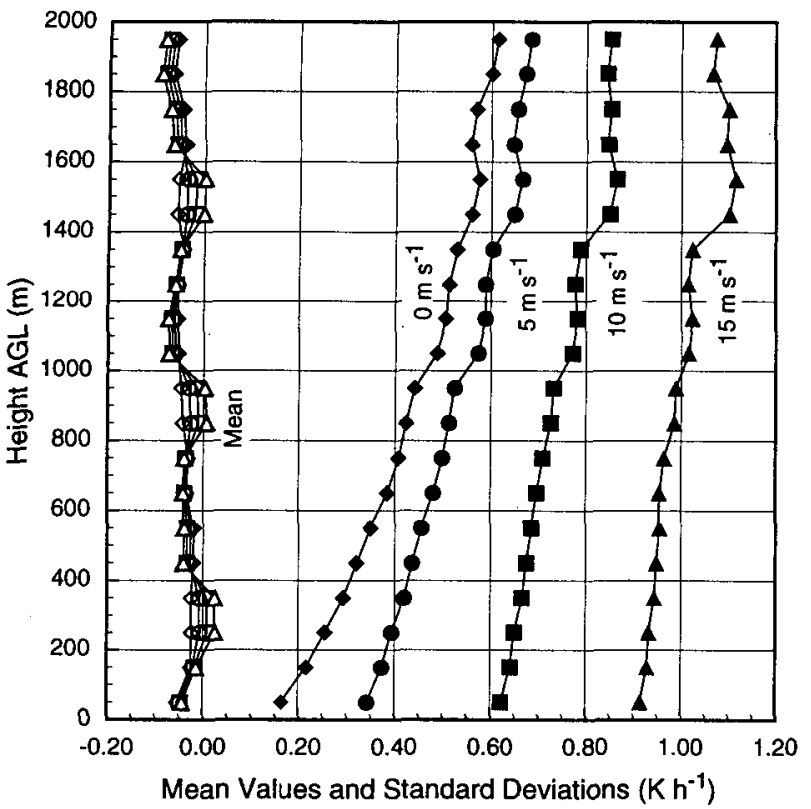

FiG. 3. As in Fig. 2 but for mean wind speeds of $0,5,10$, and 15 $\mathrm{m} \mathrm{s}^{-1}$. The measurement errors are the same as for the control run. Statistics computed from 1000 Monte Carlo simulations, $0 \mathrm{~m} \mathrm{~s}^{-1}$ $(\bullet) ; 5 \mathrm{~m} \mathrm{~s}^{-1}(\bullet) ; 10 \mathrm{~m} \mathrm{~s}^{-1}(\square) ;$ and $15 \mathrm{~m} \mathrm{~s}^{-1}(\Delta)$.

erably less than the observed magnitude of typical convective boundary layer turbulent sensible heat flux divergences.

At present RASS temperatures are typically measured only for the last $5 \mathrm{~min}$ of each hour. Increasing the total measurement time for RASS by a factor of 2 or 3, while sampling at shorter intervals spaced throughout the hour, would significantly reduce the temperature sampling errors. Because a short decrease in the sampling time for winds would not seriously degrade their accuracy, and because the sensitivity to $\delta \mathbf{v}$ is less than that to $\delta \theta$ (Figs. 2 and 4 ), a net improvement in the accuracy of the heat budget would result. Note that the effects discussed here relate only to random errors, not to systematic errors such as described in Angevine and Ecklund (1994).

\section{e. Sensitivity to mean stability}

The mean atmospheric stability enters the residual error through term Bvm. From the control run and our scaling analysis we see that this term is relatively important, and that it is the more important of the two terms (Bvm and Bvp) that have a height dependence (other than density effects in term $\mathrm{D}$ ). By reducing the stability toward neutral, we reduce the slope of the residual error while the surface intercept remains unchanged, as shown in Fig. 5. A comparison with Fig. 3 shows that for the selected parameter ranges, the sensitivity to mean stability and mean wind are of similar magnitude.

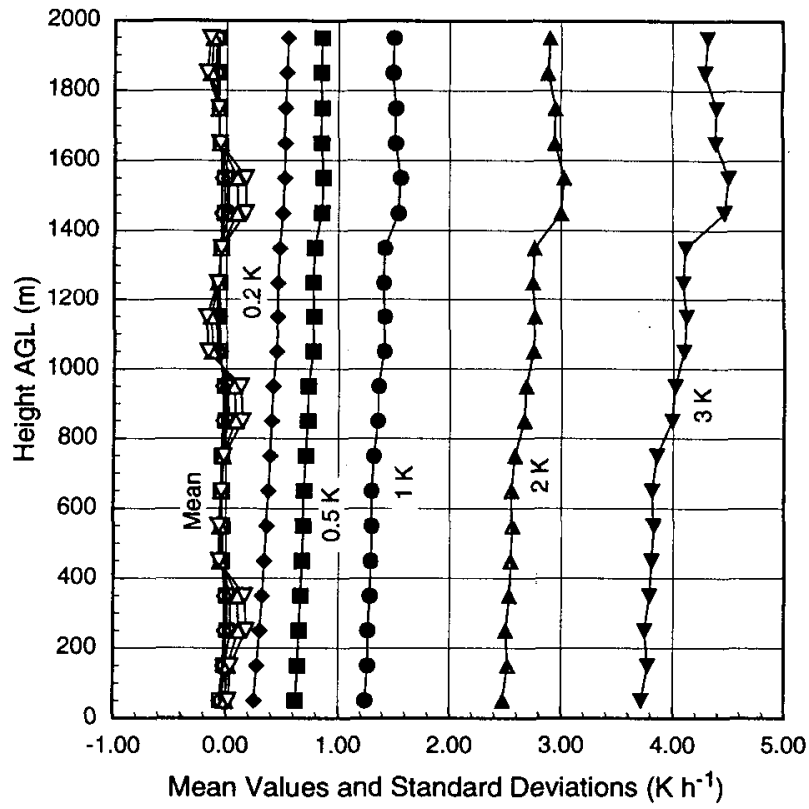

FIG. 4. As in Fig. 2 but for temperature measurement errors of 0.2 , $0.5,1,2$, and $3 \mathrm{~K}$ instead of wind measurement errors; $0.2 \mathrm{~K}(\bullet)$; $0.5 \mathrm{~K}(\mathbf{G}) ; 1 \mathrm{~K}(\bullet) ; 2 \mathrm{~K}(\boldsymbol{\Delta})$; and $3 \mathrm{~K}(\boldsymbol{\nabla})$.

\section{f. Effects of radiation errors}

Figure 6 shows the results of simulations of net radiative flux divergence for net radiation measurement errors of $1.5,5$, and $15 \mathrm{~W} \mathrm{~m}^{-2}$, respectively. We find

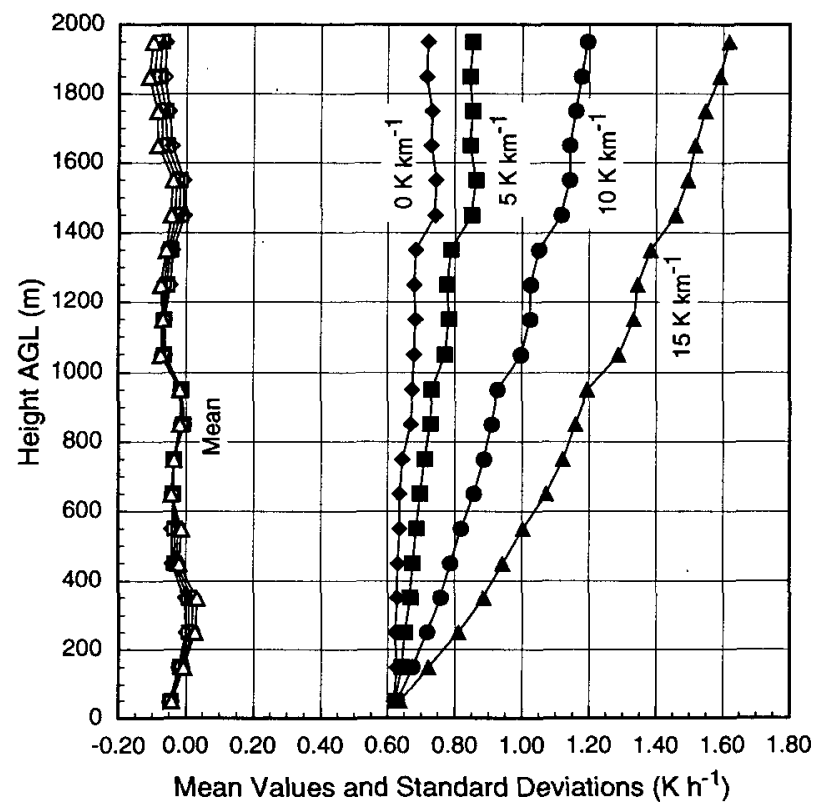

FIG. 5. As in Fig. 2 but for mean stability values $\partial \theta / \partial z$ of $0,5,10$, and $15 \mathrm{~K} \mathrm{~km}^{-1}$ instead of wind measurement errors; $0 \mathrm{~K} \mathrm{~km}^{-1}(\diamond)$; $5 \mathrm{~K} \mathrm{~km}^{-1}(\boldsymbol{\Xi}) ; 10 \mathrm{~K} \mathrm{~km}^{-1}(\bullet)$; and $15 \mathrm{~K} \mathrm{~km}^{-1}(\Delta)$. 


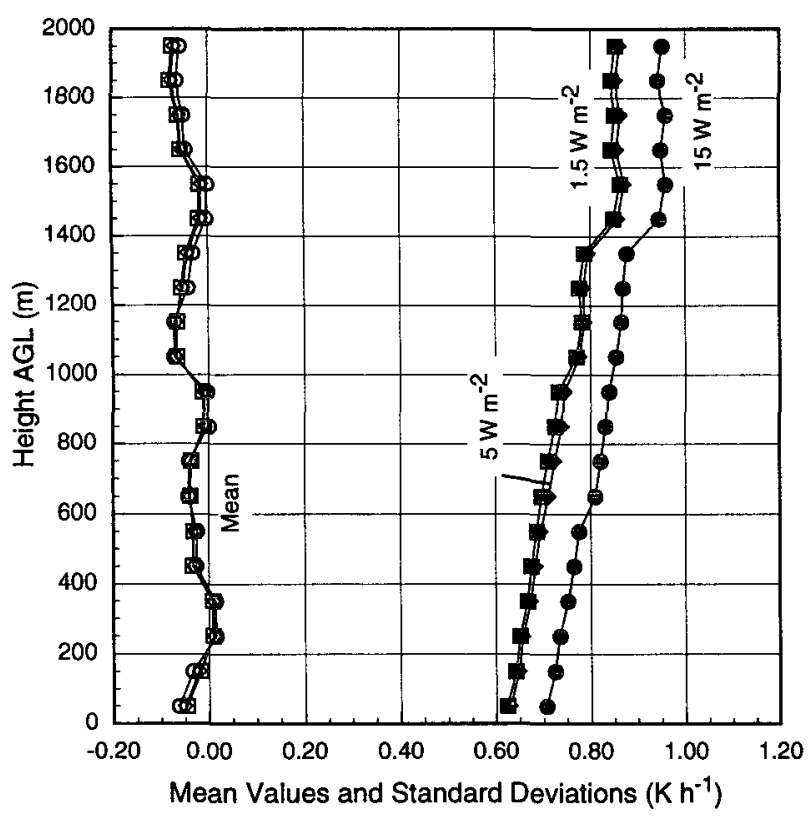

Fig. 6. As in Fig. 2 but for net radiative flux measurement errors of $1.5,5$, and $15 \mathrm{~W} \mathrm{~m}^{-2}$ instead of wind measurement errors; 1.5 $\mathrm{W} \mathrm{m} \mathrm{m}^{-2}(\mathbf{D}) ; 5 \mathrm{~W} \mathrm{~m}^{-2}(\bullet)$; and $15 \mathrm{~W} \mathrm{~m}^{-2}(\bullet)$.

only a minor difference of about $10 \%$ between the curves for 5 and $15 \mathrm{~W} \mathrm{~m}^{-2}$. In calm situations with weak turbulence, radiative cooling may become the key process in the heat budget. In such situations, the standard deviations of term $\mathrm{C}$ will be of the order of 0.5 $\mathrm{K} \mathrm{h}^{-1}$ (see Fig. 3) and may be comparable to values of radiative cooling calculated by Garratt and Brost (1981) for the surface layer $\left(-1 \mathrm{~K} \mathrm{~h}^{-1}\right)$ and for the layer immediately above the surface layer $(-0.2$ $\mathrm{K} \mathrm{h}^{-1}$ ).

Because we are using climatological values of radiative flux divergences as obtained over rather deep atmospheric layers, we consider the issue of the radiation profile an open question that will be left for future analysis.

\section{g. Time averaging and geographic effects}

The previous simulations were performed for 1-h averages, but the standard deviation of term $\mathrm{C}$ could be reduced by increasing the sample size (i.e., averaging time). If the meteorological fields were stationary, for example, a sample of $n$ 1-h averages would reduce the standard deviation by a factor $n^{-1 / 2}$, although the factor would generally be less because of stationarity assumption violations. The results of simulations for averaging periods of 1,3 , and $6 \mathrm{~h}$ for the control run are shown in Fig. 7. The effect is a decrease of uncertainty proportional to $n^{-1 / 2}$. Long averaging periods are, however, of limited applicability to dynamic meso- $\gamma$ scale studies because the diurnal variation cannot be adequately resolved. This is due either to excessive

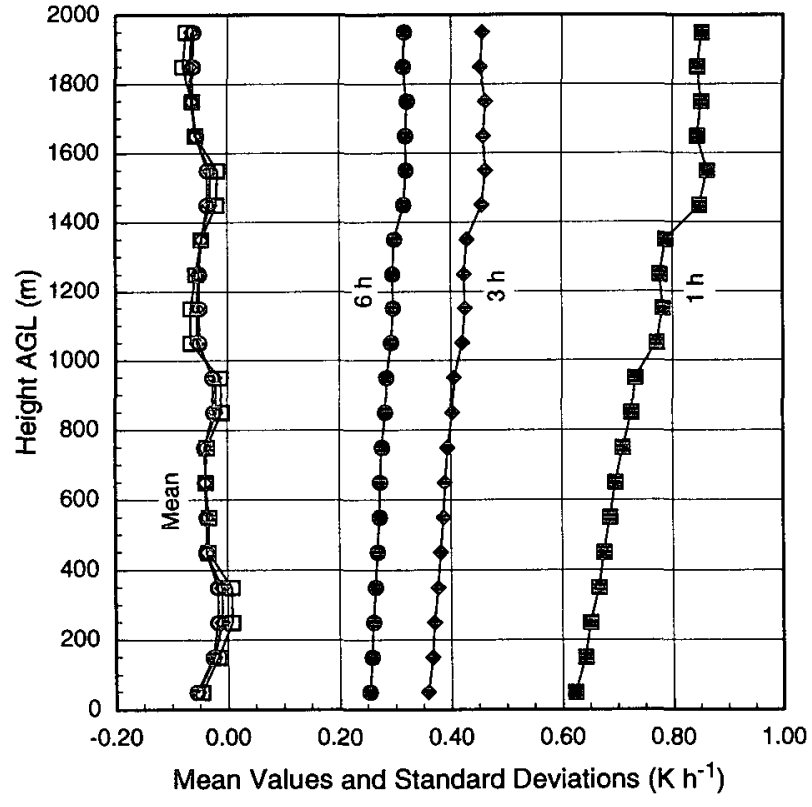

Fig. 7. Mean values (denoted as "mean," outline symbols) and standard deviations (solid symbols) of the turbulent potential temperature flux divergence (term $\mathrm{C}$ ), calculated as a residual of the other terms, for averaging times of 1,3 , and $6 \mathrm{~h} ; 1 \mathrm{~h}(\mathbf{D}) ; 3 \mathrm{~h}(\bullet)$; and 6 $\mathrm{h}(\bullet)$.

smoothing in the case of averaging several consecutive hours or to large synoptic variability in the case of averaging the same time of day for several consecutive days. On the other hand, useful results can be expected

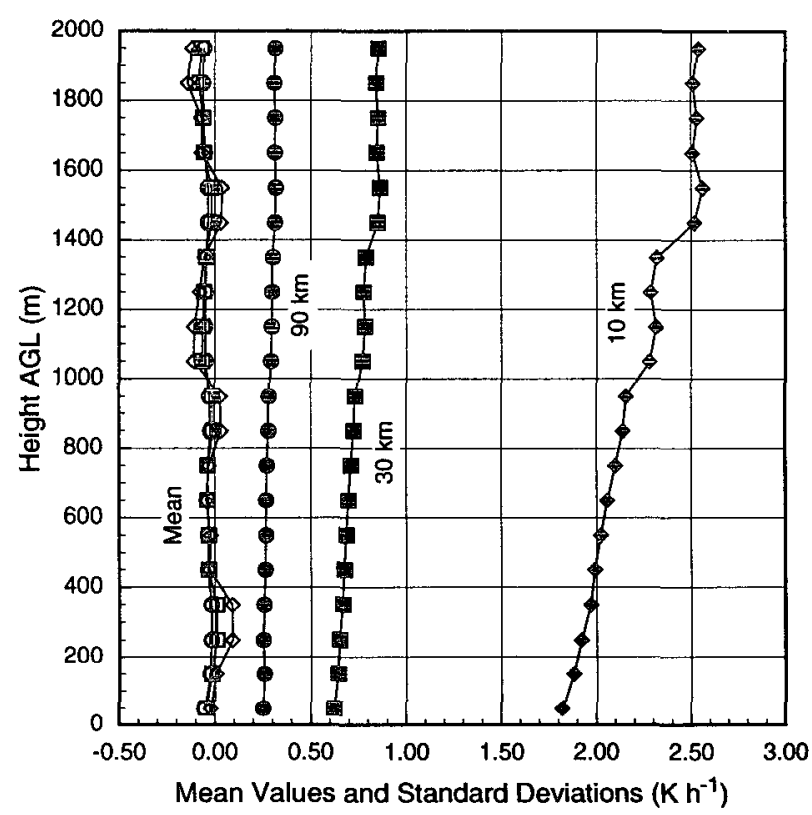

Fig. 8. As in Fig. 7 but for triangle side lengths of 10,30, and $90 \mathrm{~km} ; 10 \mathrm{~km}(\bullet) ; 30 \mathrm{~km}(\mathbf{0})$; and $90 \mathrm{~km}(\bullet)$ 
for a long-term climatology, for example, monthly mean values (May and Wilczak 1993).

Another important issue is the effect of the size of the triangle. Figure 8 shows results of three simulations for side lengths of 10,30 , and $90 \mathrm{~km}$. Measurement errors were the same as in the control run. The resulting standard deviations decrease with increasing triangle size to reach values of $0.3 \mathrm{~K} \mathrm{~h}^{-1}$ for $90 \mathrm{~km}$. This is a smoothing effect that arises from the fact that wind and temperature gradients along the triangle sides become smaller for longer side lengths provided the measured quantities remain the same.

\section{Conclusions}

Our study investigated the uncertainty in the calculation and evaluation of the heat (or, more precisely, the virtual potential temperature) budget equation in the atmospheric boundary layer from data collected with $915-\mathrm{MHz}$ WP-RASS. A scaling analysis of the residual error heat budget equation yielded information on the magnitudes and general behavior of the uncertainties of individual budget terms. Subsequently, a Monte Carlo technique was used to verify the estimated uncertainties of individual terms under idealized conditions. The results for the simulated cases agreed well with those from the scaling analysis.

The heat budget was evaluated for a hypothetical 30$\mathrm{km}$ triangle of stations in ideally flat terrain. To test the influence of measurement uncertainties on the budget calculations, a stationary, horizontally homogeneous meteorological situation was assumed. Net radiative flux divergence was estimated from climatological data and a radiative transfer model. Turbulent virtual potential temperature flux divergence was evaluated as a heat budget equation residual. Given 1 -h average relative instrument accuracies of $0.5 \mathrm{~K}, 1 \mathrm{~m} \mathrm{~s}^{-1}$, and 1.5

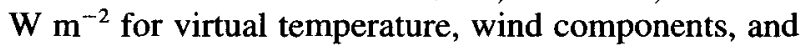
net radiation, respectively, and a vertical resolution of $100 \mathrm{~m}$, this term can be calculated with a relative accuracy of $0.4-1.6 \mathrm{~K} \mathrm{~h}^{-1}$, depending on mean wind speed and stability. Given the assumptions of the surface-integral method, these values are an initial estimate of uncertainty resulting from measurement errors. Because there are other sources of uncertainty, we cannot provide estimates of overall uncertainty. Among these other sources is the violation of the assumption of linear variation of the meteorological fields. An improvement of the algorithm for the calculation of the horizontal wind divergence, perhaps by including vertical wind components measured by the profiler, would possibly reduce, but not erase, the increase of uncertainty with height in divergent situations.

The Monte Carlo simulations show that individual terms of the budget equation are not equally sensitive to measurement errors. Improvements in the evaluation of the heat budget equation would accrue from improvements in temperature measurement accuracy and, to a lesser extent, from improvements in wind measurement accuracy, at least in weakly divergent flows. The simulations revealed a dependence of the uncertainties on measurement errors, atmospheric stability, mean wind speed, averaging times, triangle size, baroclinity, and wind divergence. The mean virtual potential temperature flux divergence term (advection) was the most sensitive to measurement errors. Calculation of the turbulence term as a residual prevented a complete evaluation of the uncertainty of the surface-integral method. However, the Monte Carlo simulations proved to be a very useful, yet conceptually simple, tool for performing uncertainty analyses. It can be applied to individual case studies and will produce appropriate uncertainty evaluations for individual terms in the heat budget equation. The results of the Monte Carlo simulations suggest that, under favorable conditions, reasonably accurate estimates of each of the terms of the atmospheric heat budget (excluding radiation) can be derived for the ABL from measurements obtained with a triangular array of ground-based remote sensors.

An application of the method to real data was not yet possible because no suitable dataset of WP-RASS measurements was available at this time.

Wind profilers with RASS provide nearly continuous measurements of the advection term so often neglected in ABL heat budget studies. Summertime experiments seem more promising with respect to the height coverage of the RASS data, as RASS measurements go higher in warm, humid air. The need for deep, unbroken, high-quality data records from remote sensing networks should be emphasized. Further refinement is required for the proper evaluation of the radiative flux divergence term. In addition, baroclinity and wind divergence effects remain for further investigation, but our rough estimates suggest that in strongly baroclinic and/or divergent situations significant errors in the estimate of the heat budget will occur.

Acknowledgments. M. Furger wishes to thank the U.S. Department of Energy's (DOE's) Atmospheric Studies in Complex Terrain (ASCOT) and Atmospheric Radiation Measurements (ARM) programs for supporting his one-year Adjunct Environmental Scientist appointment at Washington State University spent at Pacific Northwest Laboratory (PNL). He also wishes to thank K. J. Allwine at PNL for programming assistance.

M. Furger and C. D. Whiteman wish to acknowledge funding support from DOE under Contract DE-AC0676RLO 1830 at PNL. PNL is operated for DOE by Battelle Memorial Institute.

\section{REFERENCES}

Angevine, W. M., and W. L. Ecklund, 1994: Errors in radio acoustic sounding of temperature. J. Atmos. Oceanic Technol., 11, 4249. 
D. A. Carter, K. S. Gage, and K. P. Moran, 1994: Improved radio acoustic sounding techniques. J. Atmos. Oceanic Technol, 11, 837-842.

Binder, P., H. C. Davies, and J. Horn, 1989: Free atmosphere kinematics above the northern Alpine foreland during the ALPEXSOP. Contrib. Atmos. Phys., 62, 30-45.

Carlson, C. A., and G. S. Forbes, 1989: A case study using kinematic quantities derived from a triangle of VHF Doppler wind profilers. J. Atmos. Oceanic Technol., 6, 769-778.

Carlson, M. A., and R. B. Stull, 1986: Subsidence in the nocturnal boundary layer. J. Climate Appl. Meteor., 25, 1088-1099.

Carter, D. A., W. L. Ecklund, K. S. Gage, M. Spowart, H. L. Cole, E. F. Chamberlain, W. F. Dabberdt, and J. Wilson, 1992: First test of a shipboard wind profiler. Bull. Amer. Meteor. Soc., 73, 1587-1592.

Davies-Jones, R., 1993: Useful formulas for computing divergence, vorticity, and their errors from three or more stations. Mon. Wea. Rev., 121, 713-725.

Garratt, J. R., and R. A. Brost, 1981: Radiative cooling effects within and above the nocturnal boundary layer. J. Atmos. Sci., 38, $2730-2746$.

Gaynor, J. E., and J. P. Ye, 1993: Simulation of RASS temperatures using fast response temperature sensors on a tall tower. Preprints, Eighth Symp. on Meteorological Observations and Instrumentation, Anaheim, CA, American Meteorological Society, $165-168$.

Hermes, L. G., 1991: Comparison of rawinsonde-deduced kinematic and thermodynamic quantities with those deduced from VHF profiler observations. Mon. Wea. Rev., 119, 1693-1712.

Hoehne, W. E., 1980: Precision of National Weather Service upper air measurements. NOAA Tech. Memo., NWS T\&ED-16, 23 pp.

Kondratyev, K. Y., 1972: Radiation processes in the atmosphere. WMO No. 309, 214 pp.

Lataitis, R. J., R. G. Strauch, and K. P. Moran, 1993: Temperature errors in RASS caused by winds and turbulence. Preprints, 26 th Int. Conf. on Radar Meteorology, Norman, OK, American Meteorological Society, 614-616.

Martner, B. E., D. B. Wuertz, B. B. Stankov, R. G. Strauch, E. R. Westwater, K. S. Gage, W. L. Ecklund, C. L. Martin, and W. F. Dabberdt, 1993: An evaluation of wind profiler, RASS, and microwave radiometer performance. Bull. Amer. Meteor. Soc., 74, $599-613$.

May, P. T., and J. M. Wilczak, 1993: Diurnal and seasonal variations of boundary-layer structure observed with a radar wind profiler and RASS. Mon. Wea. Rev., 121, 673-682.

$\longrightarrow$, K. P. Moran, and R. G. Strauch, 1989: The accuracy of RASS temperature measurements. J. Appl. Meteor., 28, 1329-1335.

McKee, T. B., and C. D. Whiteman, 1977: Components of infrared net radiation in a mountain valley. Atmospheric Science Pa- per No. 279, Colorado State University, Fort Collins, CO, $102 \mathrm{pp}$.

Moran, K. P., and R. G. Strauch, 1994: The accuracy of RASS temperature measurements corrected for vertical air motion. $J$. Atmos. Oceanic Technol., 11, 995-1001.

- , D. B. Wuertz, R. G. Strauch, N. L. Abshire, and D. C. Law, 1991: Temperature sounding with wind profiler radars. $J$. Atmos. Oceanic Technol., 8, 606-608.

Neff, W. D., and J. M. Wilczak, 1992: The application of $915-\mathrm{MHz}$ radar wind profilers to the study of transport in the atmospheric boundary layer. Proc. Tenth Symp. Turbulence and Diffusion, Portland, OR, American Meteorological Society, 276-280.

Pielke, R. A., 1984: Mesoscale Meteorological Modeling. Academic Press, 612 pp.

Press, W. H., B. P. Flannery, S. A. Teukolsky, and W. T. Vetterling, 1989: Numerical Recipes: The Art of Scientific Computing (Fortran Version). Cambridge University Press, 702 pp.

Saarikivi, P., and T. Puhakka, 1990: The structure and evolution of a wintertime occluded front. Tellus, 42A, 122-139.

Strauch, R. G., D. A. Merritt, K. P. Moran, K. B. Earnshaw, and D. van de Kamp, 1984: The Colorado wind-profiling network. $J$. Atmos. Oceanic Technol., 1, 37-49.

$\longrightarrow$ B. L. Weber, A. S. Frisch, C. G. Little, D. A. Merritt, K. P. Moran, and D. C. Welsh, 1987: The precision and relative accuracy of profiler wind measurements. J. Atmos. Oceanic Technol., 4, 563-571.

Thiébaux, H. J., and M. A. Pedder, 1987: Spatial Objective Analysis. Academic Press, 299 pp.

Weber, B. L., and D. B. Wuertz, 1990: Comparison of rawinsonde and wind profiler radar measurements. J. Atmos. Oceanic Technol., 7, 157-174.

, R. G. Strauch, D. A. Merritt, K. P. Moran, D. C. Law, D. van de Kamp, R. B. Chadwick, M. H. Ackley, M. F. Barth, N. L. Abshire, P. A. Miller, and T. W. Schlatter, 1990: Preliminary evaluation of the first NOAA demonstration network wind profiler. J. Atmos. Oceanic Technol., 7, 909-918.

, — D. C. Law, A. S. Frisch, and J. M. Brown, 1992: Effects of small-scale vertical motion on radar measurements of wind and temperature profiles. J. Atmos. Oceanic Technol., 9, 193209.

Wuertz, D. B., B. L. Weber, R. G. Strauch, A. S. Frisch, C. G. Little, D. A. Merritt, K. P. Moran, and D. C. Welsh, 1988: Effects of precipitation on UHF wind profiler measurements. J. Atmos. Oceanic Technol., 5, 450-465.

Ye, J. P., D. E. Wolfe, J. E. Gaynor, and D. C. Welsh, 1993: A detailed comparison between wind profiler and tower measurements. Preprints, Eighth Symp. on Meteorological Observations and Instrumentation, Anaheim, CA, American Meteorological Society, 298-303. 